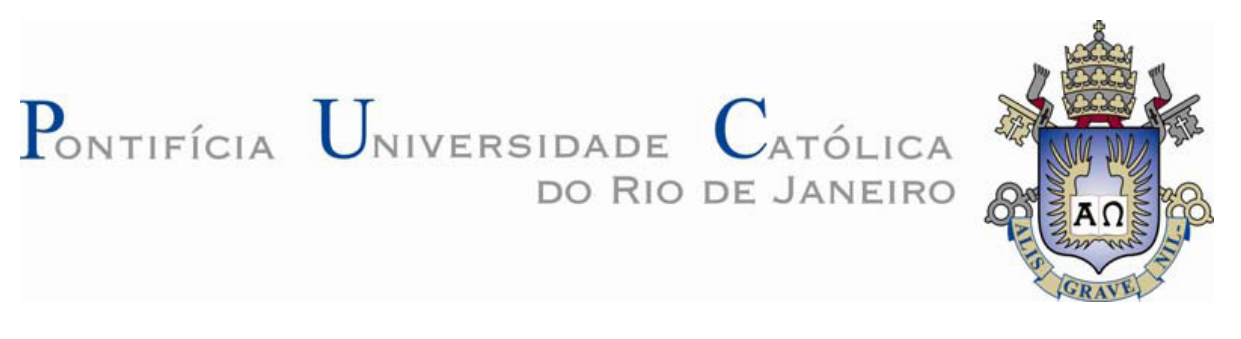

Jose Luis Montalvo Andia

Caracterização Microestrutural, Mecânica e Simulação

Física da ZTA em Aço API X80

Dissertação apresentada como requisito parcial para obtenção do título de Mestre pelo Programa de Pós-Graduação em Engenharia de Materiais e de Processos Químicos e Metalurgicos do Departamento de Engenharia de Materiais da PUCRio.

Orientador: Profa. Ivani de Souza Bott 


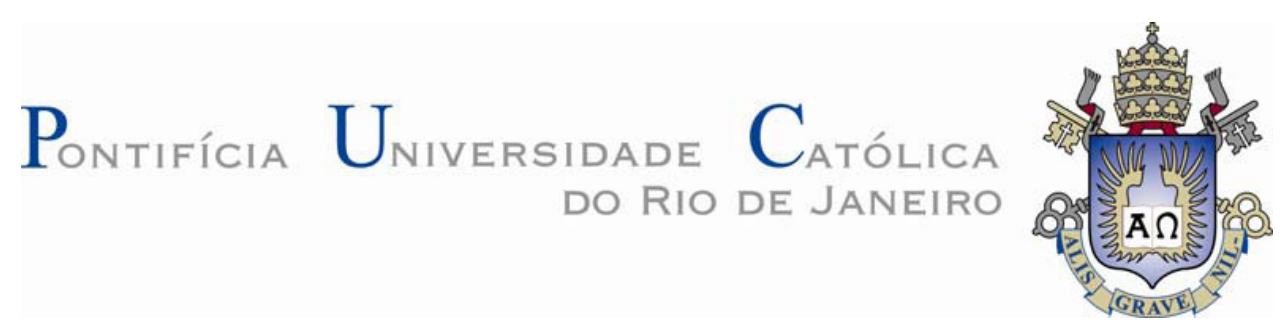

Jose Luis Montalvo Andia

\section{Caracterização Microestrutural, Mecânica e Simulação \\ Física da ZTA em Aço API X80}

Dissertação apresentada como requisito parcial para obtenção do título de Mestre pelo Programa de PósGraduação de Engenharia de Materiais e de Processos Químicos e Metalúrgicos do Departamento de Engenharia de Materiais da PUC-Rio. Aprovada pela Comissão Examinadora abaixo assinada.

Profa. Ivani de Souza Bott Orientador Departamento de Engenharia de Materiais - PUC-Rio

Prof. Antonio José Ramírez Londono Laboratório Nacional de Nanotecnologia de Brasil - LNNano/SP

Prof. Luís Felipe G. de Souza Centro Federal de Educação Tecnológica Celso Suckow FonsecaCEFET/RJ

Prof. Valter Rocha dos Santos Departamento de Engeharia de Materiais- PUC-Rio

Prof. José Eugênio Leal Coordenador Setorial de Pós-Graduação do Centro Técnico Científico da PUC-Rio 
Todos os direitos reservados. É proibida a reprodução total ou parcial do trabalho sem autorização da universidade, do autor e do orientador.

\section{Jose Luis Montalvo Andia}

Graduou-se em Engenharia Metalúrgica na Universidade Nacional de San Agustín de Arequipa, no Perú em (2009). Possui experiência profissional como Engenheiro na área de inspeção de Soldagem, controle de qualidade de produtos soldados além de ter experiência em operações e processos metalúrgicos de cobre, prata e ouro.

Ficha Catalográfica

Andia, Jose Luis Montalvo

Caracterização microestrutural, mecânica e simulação física da ZTA em aço API X80 / Jose Luis Montalvo Andia ; orientadora: Ivani de Souza Bott. 2012.

135 f. : il. (color.) ; $30 \mathrm{~cm}$

Dissertação (mestrado)-Pontifícia Universidade Católica do Rio de Janeiro, Departamento de Engenharia de Materiais, 2012.

Inclui bibliografia

1. Engenharia de materiais - Teses. 2. Aços API 5L grau X80. 3. Zona termicamente afetada. 4. Caracterização microestrutural. 5. Simulação térmica. 6. Tenacidade de impacto Charpy. I. Bott, Ivani de Souza. II. Pontifícia Universidade Católica do Rio de Janeiro. Departamento de Engenharia de Materiais. III. Título.

CDD: 620.11 
A meus pais Constantino Montalvo e Vilma Marcelina Andia de Montalvo e irmôs Javier, Rildo, Jesus, Sandra pela motivação, apoio e estímulo na busca de novos conhecimentos. 


\section{Agradecimentos}

Agradeço a Deus pela saúde, fortaleza e paciência.

À minha orientadora e amiga, professora Ivani de S. Bott, pelo estimulo, ensinamentos, dedicação e amizade durante a realização deste trabalho.

Ao colega Rafael de Araujo Silva, pelo apoio e contribuições técnico-científicas.

Agradeço ao Laboratório Nacional de Nano Tecnologia (LNNano)- Campinas pela possibilidade de realização das simulações no equipamento Gleeble.

Aos engenheiros do Laboratório Nacional de Nanotecnologia Leonardo Wu e Thaís Alonso pelo apoio concedido.

Agradeço aos funcionários da PUC-Rio, em especial ao Heitor Nuss Guimarães.

Agradeço aos meus colegas da PUC-Rio, Patricia Pontón, Julio Suni, Natasha, Adriana, Fredy, Jorge e José pela ajuda e apoio durante a elaboração desta dissertação.

Agradeço a minha mãe e pai por ter me incentivado e por não medir esforços em me ajudar.

Agradeço a meu irmão Javier pelo apoio e conselhos e por estar sempre ao meu lado.

A todos os professores e funcionários do DCMM, pelos ensinamentos e ajuda.

A Capes, FAPERJ, pelos auxílios concedidos, sem os quais este trabalho não poderia ter sido realizado. 


\section{Resumo}

Andia, Jose Luis Montalvo; Bott, Ivani de Souza. Caracterização Microestrutural, Mecânica e Simulação Física da ZTA em Aço APIX80. Rio de Janeiro, 2012. 135p. Dissertação de Mestrado Departamento de Ciência dos Materiais e Metalurgia, Pontifícia Universidade Católica do Rio de Janeiro.

Foram utilizados dois sistemas de aço API 5L X80, Nb-Cr e Nb-Cr-Mo, para obter as diferentes regiões da ZTA pertencentes a uma soldagem multipasse. Estas regiões são denominadas de: região de grãos grosseiros inalterados (RGGI), região de grãos refinados reaquecidos supercriticamente (RGRRS), região de grãos grosseiros reaquecidos intercriticamente (RGGRI), região de grãos grosseiros reaquecidos subcriticamente (RGGRS). Estas regiões foram obtidas para dois aportes de calor (1,2 e 2,5 kJ/mm) e a RGGRI por ser considerada a região onde poderiam ser formadas zonas frágeis localizadas (ZFL) foram utilizados também aportes de calor de 3,0 e 4,0 kJ/mm. Cada uma das regiões obtidas pela simulação física foi submetida a ensaios mecânicos de impacto Charpy e dureza, assim como a análises metalográficos por microscopia ótica (MO) e microscopia eletrônica de varredura (MEV). Foi possível observar que as microestruturas pertencentes a uma ZTA simulada obtidas com o equipamento (Gleeble ${ }^{\circledR 3800)}$ se mostram compatíveis com aquelas pertencentes a uma soldagem real. Este resultado comprova que as velocidades de resfriamento obtidas pela simulação foram similares àquelas da soldagem real.

A adição de Mo ao sistema $\mathrm{Nb}-\mathrm{Cr}-\mathrm{Mo}$ não promoveu mudanças significativas tanto a nível microestrutural, observado por MO e MEV, como em termos de propriedades mecânicas.

\section{Palavras-chave}

Aços API 5L Grau X80; Zona Termicamente Afetada; Caracterização Microestrutural; Simulação Térmica; Tenacidade de Impacto Charpy. 


\section{Abstract}

Andia, Jose Luis Montalvo; Bott, Ivani de Souza (Advisor). API X80 HAZ Physical Simulation and Microstructural and Mechanical Characterization. Rio de Janeiro, 2012. 135p. MSc. Dissertation Departamento de Ciência dos Materiais e Metalurgia, Pontifícia Universidade Católica do Rio de Janeiro.

Two API 5L steels grade X80 of the systems $\mathrm{Nb}-\mathrm{Cr}$ and $\mathrm{Nb}-\mathrm{Cr}-\mathrm{Mo}$, were submitted to physical simulation in order to obtain different regions of the HAZ similar to those of a multipass welding, the coarse grained heat affected zone (CGHAZ), supercritically coarse grained heat affected zone (SCCGHAZ), intercritically coarse grained heat affected zone (ICCGHAZ), subcritically coarse grained heat affected zone (SCGHAZ). The welding simulation was carried out on a Gleeble ${ }^{\circledR} 3800$ considering two thermal cycles and different heat inputs 1.2, 2.5, 3.0 and 4,0 kJ/mm, typical of a girth weld. All HAZ zones were simulated only for 1.2 and $2.5 \mathrm{~kJ} / \mathrm{mm}$. Since the ICCGHAZ is the probable weak link where a local brittle zone (LBZ) can occur, this region was simulated for all heat inputs studied. All simulated regions were subjected to traditional mechanical tests such as impact Charpy- $\mathrm{V}$ at -40 and $-60^{\circ} \mathrm{C}$ and microhardness $\mathrm{Hv}_{1 \mathrm{~kg}}$. Metallographic analysis by optical microscopy (OM) and scanning electron microscopy (SEM) and fractography were also performed. The microstructures obtained for the different regions of the HAZ, by simulation were close to those of a real welding, however, the cooling rates obtained by simulation were slower than that obtained in a real welding. The mechanical properties and microstructure of the different regions of the HAZ for the systems $\mathrm{NbCr}$ and $\mathrm{NbCrMo}$ indicate that the microstructural and mechanical behavior of the intercritical region (ICCGHAZ) was considered to be similar to a local brittle zone (LBZ) for all conditions studied.

\section{Keywords}

Steels API 5L Grade X80; Heat Affected Zone (HAZ); Microstructural Characterization; Thermal Simulation; Impact Touhness Charpy. 


\section{Sumário}

1 Introdução 20

$\begin{array}{ll}\text { 1.1. Objetivos } & 21\end{array}$

2 - Revisão Bibliográfica $\quad 22$

2.1. Aços de alta resistência e baixa liga (ARBL) 22

2.1.1. Evolução de aços de alta resistência e baixa liga (ARBL) 23

2.1.2. Fabricação de aços de alta resistência e baixa liga (ARBL) 24

2.2. Soldagem de aços de alta resistência e baixa liga (ARBL) 25

2.3. Fatores que afetam a tenacidade da ZTA em aços microligados 27

2.3.1. A solubilidade dos elementos microligantes: 27

2.3.2. Efeito dos elementos microligantes nas temperaturas de $\begin{array}{ll}\text { transformação } & 29\end{array}$

2.3.3. Efeito de elementos de liga no tamanho de grão austenítico da ZTA

2.4. Influência dos elementos microligantes na ZTA 33

2.4.1. Efeito de nióbio na tenacidade da ZTA 33

2.4.2. Influência do nitrogênio na tenacidade da ZTA 35

2.4.3. Efeito de vanádio na tenacidade da ZTA 36

2.4.4. Efeito do titânio na tenacidade da ZTA 37

2.4.5. Efeito de molibdênio na tenacidade da ZTA 38

2.4.6. Carbono equivalente (CE) 39

2.5. Simulações de soldagem 40

2.5.1. Gleeble $3800 \quad 41$

2.5.2. Modelos da Gleeble® 3800

2.5.3. Ciclo térmico 44

2.5.4. Parâmetros que influenciam o ciclo térmico de soldagem 45

2.5.4.1. Temperatura de preaquecimento 45

2.5.4.2. Aporte de calor 46

$\begin{array}{ll}\text { 2.5.4.3. Temperatura pico } & 47\end{array}$ 
2.5.4.4. Tempo de resfriamento $(\Delta t 8 / 5) \quad 49$

2.6. Zona Termicamente Afetada (ZTA) 50

2.6.1. Característica da ZTA de uma solda de passe único. 50

2.6.2. Características da ZTA de uma solda multipasse 52

2.6.3. Influência de zonas frágeis localizadas (ZFL) na ZTA 54

2.6.3.1. Principais mecanismos que influenciam a (ZFL) 54

3 - Metodologia Experimental 56

3.1. Descrição do método experimental 56

3.2. Características das amostras empregadas na simulação 57

3.3. Características das amostras empregadas na soldagem real 58

3.4. Parâmetros de simulação de soldagem 59

3.5. Parâmetros da soldagem real 60

3.6. Execução da simulação 60

3.7. Execução da solda real 62

3.8. Codificação para a avaliação da ZTA 63

3.9. Análise Metalográfica 64

3.9.1. Microscopia Ótica (MO) 65

3.9.2. Microscopia Eletrônica de varredura (MEV) 65

3.10. Ensaios de Impacto Charpy-V 66

3.11. Caracterização Metalográfica e Fractográfica dos corpos de $\begin{array}{ll}\text { prova Charpy } & 67\end{array}$

3.11.1. Análises de fratura interna dos corpos de prova Charpy 67

3.12. Ensaio de microdureza Vickers (HV) 68

4. Resultados 70

4.1. Determinação dos ciclos térmicos simulados 70

4.2. Cálculos do tempo $\Delta \mathrm{t} 8 / 5$ e velocidade de resfriamento 74

4.3. Estudo da Influência do aporte de calor 74

4.4. Caracterização metalográfica $\quad 77$

4.4.1. Macrografia da ZTA $\quad 77$

4.4.2. Caracterização Microestrutural do metal base 78

4.4.3. Caracterização microestrutural das regiões ZTA por MO 78 
4.5. Caracterização microestrutural do constituinte austenita martensita (AM) por MEV

4.5.1. Metal de base (MB)

4.5.2. Zona termicamente afetada (ZTA)

4.5.3. Comparação do constituinte AM entre MO e MEV 90

4.6. Ensaios de impacto 93

4.6.1. Análise fractográfica 95

4.6.2. Analise da fratura interna das trincas 103

4.7. Ensaios de microdureza Vickers (HV 1kg) nas regiões da ZTA real

4.8. Ensaios de microdureza Vickers (HV1kg) para as regiões simuladas

5 . Discussão

5.1. Evolução microestrutural

5.1.1. Região de grãos grosseiros inalterados (RGGI ZTA) $1200^{\circ} \mathrm{C}$

5.1.2. Região de grãos grosseiros refinados reaquecidos supercriticamente (RGRRS ZTA) $1000^{\circ} \mathrm{C}$

5.1.3. Região de grãos grosseiros reaquecidos intercriticamente (RGGRI ZTA) $800^{\circ} \mathrm{C}$

5.1.4. Região de grãos grosseiros reaquecidos subcriticamente (RGGRS ZTA) $600^{\circ} \mathrm{C}$ 


\section{Lista de figuras}

Figura 2-1 Relação entre a tenacidade caracterizada pela temperatura de transição obtida a $27 \mathrm{~J}$ e a tensão de escoamento [1].

Figura 2-2 Evolução dos graus API ao longo dos anos respeito a tensão de escoamento $[5,12]$

Figura 2-3 Solubilidade dos principais compostos na região de grãos grosseiros na ZTA [19], onde (Wt\%A) recebe o porcentual em massa do primeiro elemento do precipitado ( $\mathrm{Nb}$ ou $\mathrm{V}$ ou $\mathrm{Ti}$ ), e (Wt\%B) recebe o porcentual em massa do segundo elemento químico do precipitado (C ou N).

Figura 2-4 Influência do manganês (a), b) e cromo (c), (d) nas temperaturas críticas de transformação, Ac1(a) e Ac3(b) [29].

Figura 2-5 Influência de nitrogênio e titânio no tamanho de grão austenítico [32].

Figura 2-6 Influência da velocidade de aquecimento no tamanho de grão austenítico, em aços ARBL. [28]

Figura 2-7 Influência do nióbio na tenacidade a fratura em ensaios Charpy (a) e CTOD (b) realizados em aços ARBL e soldados com um aporte de calor de $2 \mathrm{~kJ} / \mathrm{mm}$.

Figura 2-8 Influencia do teor de nitrogênio na energia de impacto (a), influência do nitrogênio livre na energia de impacto (b). [40]

Figura 2-9 Influencia do Ti na temperatura de transição de impacto da ZTA, soldado com um aporte de calor de $4.5 \mathrm{KJ} / \mathrm{mm}$ [45].

Figura 2-10 Relação entre a microdureza e aporte de calor na região intercrítica da ZTA para diferentes teores de Mo [50].

Figura 2-11 Comparação microestrutural da ZTA na (RGGRI) Tp2$800^{\circ} \mathrm{C}$, para uma solda real (a) e simulada (b). 
Figura 2-12 Comparação de ciclos térmicos de soldagem desenvolvidos por três modelos de fluxo de calor, Rosenthal Rykalin 2D - Rykalin 3D [54].

Figura 2-13 Influência da temperatura de preaquecimento na dureza (a) e tenacidade (b), [67].

Figura 2-14 Influencia da temperatura de pico na tenacidade da ZTA de uma solda multipasse [76].

Figura 2-15 Presença do constituinte $\mathrm{AM}$ na região reaquecida intercrítica da ZTA na forma massiva e alongada. Aumento 200X (a), aumento 1000X(b). [77].

Figura 2-16 Influência do tempo de resfriamento na formação e decomposição do constituinte AM. [77]

Figura 2-17 Diagrama esquemático dos constituintes da ZTA [71] de um aço com $0.15 \%$ C [81].

Figura 2-18 diagrama esquemático dos constituintes da ZTA de uma solda multipasse [82]

Figura 3-1 Equipamento Gleeble $₫ 3800$.

Figura 3-2 Dimensões de corpos de prova para a simulação de soldagem.

Figura 3-3 Soldador de termopar, modelo 3S50.

Figura 3-4 Câmara interna do simulador termomecânico Gleeble $3800 \AA$, mostrando as garras de cobre (indicadas pela seta).

Figura 3-5. (a) Geometria da junta circunferencial, onde $a=3 \mathrm{~mm}, b=$ $2.5 \mathrm{~mm}, E=16 \mathrm{~mm}$; (b) sequência de passes.

Figura 3-6 Localização da extração dos corpos de prova segundo a norma API-1104

Figura 3-7 Superfície examinada nos corpos de prova simulados (a) e real (b) por MO e MEV.

Figura 3-8 Dimensões do corpo de prova Charpy $\mathrm{V}$ segundo a norma ASTM E23.

Figura 3-9 Desenho do corte realizado nos corpos de prova segundo a norma API RP2Z

Figura 3-10 Desenho esquemático dos pontos de medidas de dureza da solda real. 
Figura 3-11 Desenho esquemático dos pontos de medidas de dureza da solda simulada.

Figura 4-1. Comparação dos ciclos térmicos programados e simulados (a), ampliação de ambos ciclos (b), gerados pelo modelo de curva Rykalin 3D,

Figura 4-2 Ciclos térmicos das regiões da ZTA, (RGGI), (RGRRS), (RGGRI) (RGGRS) para (a) $1,2 \mathrm{~kJ} / \mathrm{mm}$, (b) $2,5 \mathrm{~kJ} / \mathrm{mm}$, (c) 3,0 $\mathrm{kJ} / \mathrm{mm}$, (d) 4,0 kJ/mm para o sistema $\mathrm{Nb}-\mathrm{Cr}$.

Figura 4-3 Ciclos térmicos das regiões da ZTA, (RGGI), (RGRRS), (RGGRI) (RGGRS) para 1,2 kJ/mm (a), 2,5 kJ/mm(b), 3,0 $\mathrm{kJ} / \mathrm{mm}$ (c), 4,0 kJ/mm (d) para sistema Nb-Cr-Mo.

Figura 4-4 Comparação dos ciclos térmicos simulados para diferentes aportes de calor, nos sistemas Nb-Cr-Mo (a), $\mathrm{Nb}-\mathrm{Cr}(\mathrm{b})$, na RGGRI.

Figura 4-5 influencia do aporte de calor no tempo de resfriamento $(\Delta t 8 / 5)$, em aços $\mathrm{Nb}-\mathrm{Cr}-\mathrm{Mo} \mathrm{e} \mathrm{Nb-Cr}$.

Figura 4-6 influencia do aporte de calor no tempo de resfriamento $(\Delta t 8 / 5)$, em aços Nb-Cr-Mo e Nb-Cr na (RGGRI).

Figura 4-7 Influência do aporte de calor na velocidade de resfriamento em aços $\mathrm{Nb}-\mathrm{Cr}-\mathrm{Mo}$ e $\mathrm{Nb}-\mathrm{Cr}$.

Figura 4-8 Influência do aporte de calor na velocidade de resfriamento em aços $\mathrm{Nb}-\mathrm{Cr}-\mathrm{Mo}$ e $\mathrm{Nb}-\mathrm{Cr}$ na (RGGRI).

Figura 4-9 Macrografia da ZTA real (a) e da ZTA simulada (b), realizadas por um mosaico (aumento de 20X), (nital 2\%).

Figura 4-10 Microestrutura do metal de base na condição de como recebido dos sistemas $\mathrm{Nb}-\mathrm{Cr}-\mathrm{Mo}$ (a) e $\mathrm{Nb}-\mathrm{Cr}$ (b). todas as imagens foram obtidas com aumento de 500x, (Nital 2\%).

Figura 4-11 Microestruturas das diferentes regiões da ZTA, as imagens (a), (e), (i), pertencem a (RGGl); as imagens (b), (f), (j), pertencem a (RGRRS); as imagens (c), (g), (k); pertencem a (RGGRI) e as imagens (d), (h), (l) pertencem (RGGRS) para 12 $\mathrm{kJ} / \mathrm{mm}$, imagens obtidas com aumento de $500 \mathrm{x}$, (Nital 2\%).

Figura 4-12 Microestruturas das diferentes regiões da ZTA simuladas a $2,5 \mathrm{~kJ} / \mathrm{mm}$, as imagens (a), (e), pertencem a (RGGl); 
as imagens (b), (f), pertencem a (RGRRS); para 2,5 kJ/mm, imagens obtidas com aumento de 500x, (Nital 2\%).

Figura 4-13 Microestruturas das diferentes regiões da ZTA pertencentes a $2,5 \mathrm{~kJ} / \mathrm{mm}$, as imagens (c), (g), pertencem a (RGGRI) e as imagens (d), (h), pertencem (RGGRS) para 2,5 $\mathrm{kJ} / \mathrm{mm}$, imagens obtidas com aumento de 500x, (Nital $2 \%$ ).

Figura 4-14 Microestruturas da (RGGRI) da ZTA simulada para 3,0 $\mathrm{kJ} / \mathrm{mm}$, (a) sistema Nb-Cr-Mo e (b) sistema $\mathrm{Nb}-\mathrm{Cr}$, aumento de $500 x$ nital (2\%).

Figura 4-15 Microestruturas da (RGGRI) da ZTA simulada para 4,0 $\mathrm{kJ} / \mathrm{mm}$, (a) sistema Nb-Cr-Mo e (b) sistema $\mathrm{Nb}-\mathrm{Cr}$, aumento de $500 x$ nital (2\%).

Figura 4-16 Metal base, sistema Nb-Cr-Mo (a), Nb-Cr (b). Aumento 3000X, microconstituinte AM nas formas massiva e alongada. (Lepera modificado)

Figura 4-17 Morfologias do microconstituinte AM presente nas regiões da ZTA, alongado (Tipo I), massivo (Tipo II). Aumento 20000x. (Lepera modificado)

Figura 4-18 Caracterização do microconstituinte AM nas regiões da ZTA obtidas pelo (MEV), as imagens (a), (e), (i), pertencem a (RGGl); as imagens (b), (f), (j), pertencem a (RGRRS); as imagens (c), (g), (k); pertencem a (RGGRI) e as imagens (d), (h), (l) pertencem (RGGRS) para 1,2 kJ/mm. Aumento de 3000x. (Lepera modificado).

Figura 4-19 Caracterização microestrutural das diferentes regiões da ZTA obtidas pelo (MEV), as imagens (a), (e), pertencem a (RGGI); as imagens (b), (f), pertencem a (RGRRS) para 2,5 kJ/mm. Aumento de 3000x. (Lepera modificado).

Figura 4-20 Caracterização microestrutural das diferentes regiões da ZTA obtidas pelo (MEV), as imagens (c), (g), pertencem a (RGGRI) e as imagens (d), (h), pertencem (RGGRS) para 2,5 $\mathrm{kJ} / \mathrm{mm}$. Aumento de 3000x. (Lepera modificado). 
Figura 4-21 Caracterização microestrutural do microconstituinte AM na (RGRRI) para aportes de calor de $3.0 \mathrm{~kJ} / \mathrm{mm}$, aumento $3000 \mathrm{X}$, (Lepera modificado).

Figura 4-22 Caracterização microestrutural do microconstituinte AM na (RGRRI) para aportes de calor de e $4.0 \mathrm{KJ} / \mathrm{mm}$, aumento 3000X, (Lepera modificado).

Figura 4-23 Caracterização do AM por MO e MEV das regiões, RGGI (a), (e), RGRRS (b(f)), da ZTA simulada pertencente ao sistema Nb-Cr-Mo, imagens obtidas com um aumento de (1000x), (Lepera modificado).

Figura 4-24 Caracterização do AM por MO e MEV das regiões, RGGRI (c)(g), RGGRS (d)(h), da ZTA simulada pertencente ao sistema $\mathrm{Nb}-\mathrm{Cr}-\mathrm{Mo}$, imagens obtidas com um aumento de (1000x), (Lepera modificado).

Figura 4-25 Tenacidade das diferentes regiões da ZTA caracterizadas por suas temperaturas de pico, pertencentes aos sistemas Nb-Cr-Mo e Nb-Cr para aportes de calor de (a) 1,2 kJ/mm e (b) $2,5 \mathrm{~kJ} / \mathrm{mm}$ a -40 e $-60^{\circ} \mathrm{C}$.

Figura 4-26 Influência do aporte de calor a tenacidade da RGGRI da ZTA pertencentes aos sistemas $\mathrm{Nb}-\mathrm{Cr}$-Mo e $\mathrm{Nb}-\mathrm{Cr}$ para aportes de calor de $1,2 \mathrm{~kJ} / \mathrm{mm}, 2,5 \mathrm{~kJ} / \mathrm{mm}, 3,0 \mathrm{k} / \mathrm{mm}$ e $4,0 \mathrm{~kJ} / \mathrm{mm}$, a - 40 e $60^{\circ} \mathrm{C}$.

Figura 4-27 Fractografía dos Cp's ensaiados na região (RGGRS) pertencente ao sistema $\mathrm{Nb}-\mathrm{Cr}-\mathrm{Mo}$ representando as energias máximas e mínimas a 1000x.

Figura 4-28 Fractografía dos Cp's ensaiados na região (RGGRS) pertencente ao sistema $\mathrm{Nb}-\mathrm{Cr}$ representando as energias máximas e mínimas a 1000x.

Figura 4-29 Fractografía dos Cp's ensaiados na região (RGGRI), pertencente ao sistema $\mathrm{Nb}-\mathrm{Cr}-\mathrm{Mo}$ representando as energias máximas e mínimas a 1000x.

Figura 4-30 Fractografía dos $C p$ 's ensaiados na região (RGGRI ZTA), pertencente ao sistema $\mathrm{Nb}-\mathrm{Cr}$ representando as energias máximas e mínimas a 1000x. 
Figura 4-31 Fractogáfia dos Cp's ensaiados na região (RGRRS), pertencente ao sistema $\mathrm{Nb}-\mathrm{Cr}-\mathrm{Mo}$ representando as energias máximas e mínimas a 1000x.

Figura 4-32 Fractogáfia dos Cp's ensaiados na região (RGRRS), pertencente ao sistema $\mathrm{Nb}-\mathrm{Cr}$ representando as energias máximas e mínimas a 1000x.

Figura 4-33 Fractogáfia dos Cp's ensaiados na região (RGGl), pertencente ao sistema $\mathrm{Nb}-\mathrm{Cr}-\mathrm{Mo}$ representando as energias máximas e mínimas a 1000x.

Figura 4-34 Fractogáfia dos Cp's ensaiados na região (RGGl), pertencente ao sistema $\mathrm{Nb}-\mathrm{Cr}$ representando as energias máximas e mínimas a 1000x.

Figura 4-35 trincas secundarias pertencentes a (RGGl) por microscopia ótica (MO) 500X e microscopia eletrônica de varredura (MEV) 2000X, Nital 2\%.

Figura 4-36 Trincas secundarias pertencentes a (RGGl) por microscopia ótica (MO) 500X e microscopia eletrônica de varredura (MEV) 2000X, Nital 2\%.

Figura 4-37 Trincas secundarias pertencentes a (RGGl) por microscopia ótica (MO) 500X e microscopia eletrônica de varredura (MEV) 2000X, Nital 2\%.

Figura 4-38 Mapeamento da microdureza nas diferentes regiões da ZTA para o sistema (a) Nb-Cr-Mo, (b) Nb-Cr.

Figura 4-39 Influencia do tempo de resfriamento na microdureza.

Figura 4-40 influencia da velocidade de resfriamento na microdureza.

Figura 5-1 Comparação do tempo de resfriamento (a) e velocidade de resfriamento (b) nos sistemas ( $\mathrm{Nb}-\mathrm{Cr}-\mathrm{Mo}$ e $\mathrm{Nb}-\mathrm{Cr}$ ) da RGGI para aportes de calor de 1,2 e 2,5 KJ/mm.

Figura 5-2 Comparação do tempo de resfriamento (a) e velocidade de resfriamento (b) nos sistemas (Nb-Cr-Mo e $\mathrm{Nb}-\mathrm{Cr}$ ) da RGGI para um determinado aporte de calor. 
Figura 5-3 Variação do tempo de resfriamento (a) e velocidade de resfriamento (b) nos sistemas ( $\mathrm{Nb}-\mathrm{Cr}-\mathrm{Mo}$ e $\mathrm{Nb}-\mathrm{Cr}$ ) da RGGI para um determinado aporte de calor. 


\section{Lista de tabelas}

Tabela 2-1 Temperatura de preaquecimento de acordo com as especificações técnicas para construção de plataformas fixas de aço da Petrobras [30, 69].

Tabela 3-1 Propriedades mecânicas dos aços API $5 \mathrm{~L}$ X80 Nb-CrMo e Nb-Cr.

Tabela 3-2 Composição química do aço API $5 \mathrm{~L}$ X80 do sistema NbCr-Mo e Nb-Cr.

Tabela 3-3 Composição química da chapa utilizada para a fabricação do tubo.

Tabela 3-4 Parâmetros empregados na simulação de soldagem.

Tabela 3-5 Propriedades físicas do aço API 5L X80.

Tabela 3-6 Parâmetros do processo de soldagem real.

Tabela 3-7 Relação da segunda temperatura de pico TP2 com as regiões da ZTA.

Tabela 3-8 Codificação dos corpos de prova empregados no processo de simulação e no ensaio de impacto Charpy V.

Tabela 3-9 Codificação dos corpos de prova da soldagem real

Tabela 3-10 Ataques químicos utilizados no MEV.

Tabela 3-11 Distribuição dos corpos de prova Charpy para cada região da ZTA

Tabela 4-1 Códigos das diferentes regiões da ZTA pertencente ao sistema $\mathrm{Nb}-\mathrm{Cr}$.

Tabela 4-2 Códigos das diferentes regiões da ZTA pertencentes ao sistema Nb-Cr-Mo.

Tabela 4-3 Valores meios do tempo e velocidades de resfriamento para os sistemas Nb-Cr-Mo e Nb-Cr.

Tabela 4-4 Valores médios resultantes dos ensaios de impacto Charpy-V, para as diferentes regiões da ZTA pertencentes aos sistemas $\mathrm{Nb}-\mathrm{Cr}-\mathrm{Mo}$ e $\mathrm{Nb}-\mathrm{Cr}$. 
Tabela 4-5 Resultados de microdureza do sistema (Nb-Cr-Mo)

Tabela 4-6 Valores médios da microdureza para o sistema Nb-Cr$\mathrm{Mo}$ e $\mathrm{Nb}-\mathrm{Cr}$.

Tabela 4-7 Influência do tempo de resfriamento na dureza na (RGGRI), para o sistema Nb-Cr-Mo e Nb-Cr. 